\title{
X-chromosome meiotic drive in Drosophila simulans: a QTL approach reveals the complex polygenic determinism of Paris drive suppression
}

\author{
Cécile Courret $\mathbb{B}^{1} \cdot$ Pierre R. Gérard $^{1,2} \cdot$ David Ogereau $^{1} \cdot$ Matthieu Falque $\mathbb{C}^{2} \cdot$ Laurence Moreau $^{2}$. \\ Catherine Montchamp-Moreau ${ }^{1}$
}

Received: 31 July 2018 / Revised: 14 October 2018 / Accepted: 24 October 2018 / Published online: 5 December 2018

(c) The Genetics Society 2018

\begin{abstract}
Meiotic drivers are selfish genetic elements that promote their own transmission into the gametes, which results in intragenomic conflicts. In the Paris sex-ratio system of Drosophila simulans, drivers located on the $\mathrm{X}$ chromosome prevent the segregation of the heterochromatic Y chromosome during meiosis II, and hence the production of Y-bearing sperm. The resulting sex-ratio bias strongly impacts population dynamics and evolution. Natural selection, which tends to restore an equal sex ratio, favors the emergence of resistant $\mathrm{Y}$ chromosomes and autosomal suppressors. This is the case in the Paris sex-ratio system where the drivers became cryptic in most of the natural populations of $D$. simulans. Here, we used a quantitative trait locus (QTL) mapping approach based on the analysis of 152 highly recombinant inbred lines (RILs) to investigate the genetic determinism of autosomal suppression. The RILs were derived from an advanced intercross between two parental lines, one showing complete autosomal suppression while the other one was sensitive to drive. The confrontation of RIL autosomes with a reference $X^{\mathrm{SR}}$ chromosome allowed us to identify two QTLs on chromosome 2 and three on chromosome 3, with strong epistatic interactions. Our findings highlight the multiplicity of actors involved in this intragenomic battle over the sex ratio.
\end{abstract}

\section{Introduction}

According to Mendel's law of segregation, each member of a pair of alleles in heterozygous individuals has an equal probability of being transmitted into the gametes. Alleles conferring a fitness advantage to their carriers will then be favored by natural selection. This general rule, however, suffers numerous exceptions caused by selfish genetic elements that use a variety of ways to favor their own

Electronic supplementary material The online version of this article (https://doi.org/10.1038/s41437-018-0163-1) contains supplementary material, which is available to authorized users.

Catherine Montchamp-Moreau

catherine.montchamp@egce.cnrs-gif.fr

1 Evolution Génome Comportement et Ecologie, CNRS, IRD, Université Paris-Sud, Université Paris-Saclay, Gif-sur-Yvette, 91190 Paris, France

2 Génétique Quantitative et Evolution-Le Moulon, INRA, CNRS, Université Paris-Sud, AgroParisTech, Université Paris-Saclay, Gif-sur-Yvette, 91190 Paris, France transmission at the expense of other parts of the genome and can spread even if detrimental for individual fitness. Among them, meiotic drivers pervert the process of meiosis or other steps of gametogenesis in such ways that they end up being over-represented among the functional gametes. Meiotic drive has been reported in a wide variety of organisms, including fungi, plants, insects, and mammals (Lyttle 1991; Burt and Trivers 2006; Presgraves 2009).

Sex-ratio (SR) drive is a particular type of meiotic drive where the drivers are X-linked and expressed in XY males. $\mathrm{X}$ chromosomes carrying $\mathrm{SR}$ drivers $\left(\mathrm{X}^{\mathrm{SR}}\right)$ prevent the production of functional Y-bearing sperm, which results in strong bias towards females in the offspring of carrier males. Naturally occurring cases of SR drive have now been described in 15 Drosophila species belonging to 9 different species groups or subgroups (Jaenike 2001; Zhang et al. 2015; Keais et al. 2017). The genetic and histological data indicate that the trait evolved recurrently and independently, and suggest a diversity of underlying mechanisms (Helleu et al. 2015). The ecological and evolutionary consequences of meiotic drive can be particularly far-reaching in the case of sex-linked systems. Those can trigger extended 


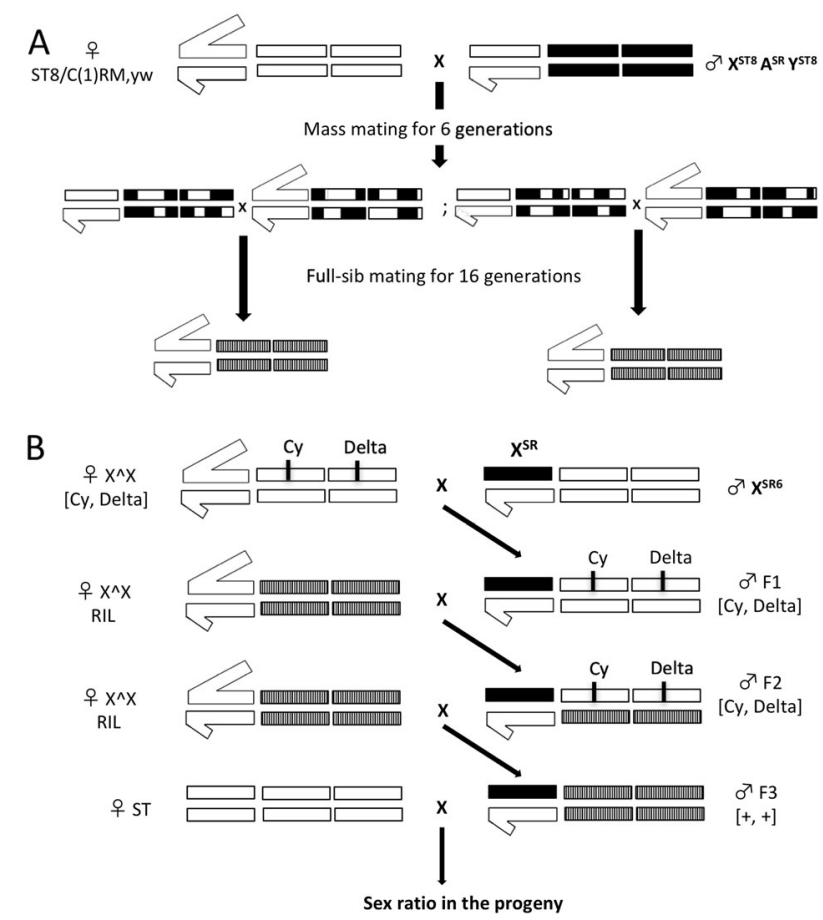

Fig. 1 a Crossing scheme used to generate the recombinant inbred lines. Standard chromosomes are shown in white and SR chromosomes are shown in black. Compound $\mathrm{X}$ are indicated by two attached boxes. The $\mathrm{Y}$ chromosome is indicated by a box with a hook. Autosomes are indicated by "A" and a simple box. Recombinant autosomes are indicated by a striped box. Large mass crossing was performed during six generations, then 600 lines were started each with a pair of F6 flies. Finally, sib-mating was performed for at least 16 generations. b Crossing scheme used to measure the suppression ability of each RIL. Standard chromosomes are shown in white and SR chromosomes are shown in black. Compound $\mathrm{X}$ are indicated by two attached boxes. The $\mathrm{Y}$ chromosome is indicated by a box with a hook. Autosomes are indicated by "A" and a simple box. Recombinant autosomes are indicated by a striped box. The black bars represent the phenotypic markers Curly and Delta, respectively on the second and third autosome. The suppression ability was determined by measuring the sex ratio in the progeny of $\mathrm{F} 3$ males

intragenomic conflict and coevolutionary arms race between the sex chromosome carrying the drivers and the rest of the genome, with impact on genome evolution, mating system, sex determination, or speciation (Helleu et al. 2015).

Unless they are hampered by very deleterious pleiotropic effects of the driver on individual fitness, $\mathrm{X}^{\mathrm{SR}}$ chromosomes can rapidly spread in populations and lead to unisexual population extinction (Hamilton 1967). However, natural selection tends to restore a balanced sex ratio and thereby favors alleles at unlinked loci when those counteract the effect of SR drivers through a negative frequencydependent selective process (Fisher 1930). When facing an $\mathrm{X}^{\mathrm{SR}}$ chromosome, a resistant $\mathrm{Y}$ chromosome, even when it is partly deleterious, has a direct and strong transmission advantage compared to sensitive $\mathrm{Y}$ chromosomes and can quickly reach fixation (Clark 1987; Unckless et al. 2015). Autosomal suppressors are favored because the rarer sex is the one that better transmits its genes: they are then selected in response to the sex-ratio bias in the population through Fisherian sex-ratio selection. It was shown in the mathematical model developed by Vaz and Carvhalo in Vaz and Carvalho (2004), that in contrast to the $Y$ chromosome, the fixation of an autosomal suppressor can occur only if the $\mathrm{X}^{\mathrm{SR}}$ is very deleterious. Such suppressor elements have been detected in many natural populations with an SR drive system. Indeed, partial autosomal suppression has evolved in Drosophila mediopunctata (Carvalho and Klaczko 1993), Drosophila paramelanica (Stalker 1961), Drosophila quinaria (Jaenike 1999), and probably in Drosophila testacea (Keais et al. 2017). In the worldwide species $D$. simulans, three SR systems have been reported; Paris, Winters, and Durham (Meiklejohn and Tao 2010). Complete suppression has evolved in all three cases in Drosophila simulans (Tao et al. 2007a; Atlan et al. 2003; Jutier et al. 2004; Tao et al. 2001), thereby making the drivers cryptic in the wild. The situation in D. simulans supports the hypothesis that $\mathrm{X}$-linked drivers are frequent in the wild, but usually "hidden" by suppressors (Hurst and Pomiankowski 1991).

With the exception of two systems in $D$. simulans, we still know very little about the molecular mechanism of SR drive and the nature of genes/DNA sequences involved in the conflict triggered by the drivers. The Winters SR system of $D$. simulans is the only case where both a SR driver and its autosomal suppressor have been identified. Dox, the driver, is a gene of unknown function, with a very limited coding capacity (Tao et al. 2007b). It was recently shown that the suppressor gene Nmy, which originated from Dox through a retrotransposition event, encodes hpRNA that repress the distorter (Lin et al. 2018). However, this putative simple model may not be representative of other SR systems. For instance, autosomal SR drive suppression in $D$. mediopunctata is multigenic, since each of the four major autosomes shows a suppressor effect (Carvalho and Klaczko 1993). Similarly, in D. simulans, the two major autosomes contribute to suppress Paris SR drive (Cazemajor et al. 1997).

The work presented here aims at further characterizing the genetic determinants of the Paris autosomal suppression, in order to better understand the evolutionary dynamics of this system where two epistatically acting Xlinked driver loci cause the missegregation of the $\mathrm{Y}$ sister chromatids during meiosis II (Montchamp-Moreau et al. 2006; Cazemajor et al. 2000). One of the drivers, has recently been identified as $H P 1 D 2$, a young and fast evolving member of the heterochromatin protein 1 (HP1) gene family (Helleu et al. 2016). The drive phenotype is caused by dysfunctional HP1D2 alleles. HP1D2 
accumulates on the heterochromatic $\mathrm{Y}$ chromosome in spermatogoniae, strongly suggesting that it directly interacts with this chromosome to control the segregation of the sister chromatids. This makes the Paris system particularly exciting as it highlights the role of genes that regulate heterochromatin structure in genetic conflicts.

We explore the genetic determinants of autosomal suppression in the Paris system by developing a quantitative trait locus (QTL) analysis based on recombinant inbred lines (RILs). The RILs were derived from an advanced intercross between two parental lines, one being highly sensitive to Paris SR drive and the other showing complete autosomal suppression. This allowed us to identify five suppression QTLs, two on chromosome 2, and three on chromosome 3 . The analysis brought to light strong epistatic interactions (i) between a major QTL on chromosome 3 and the two QTLs on chromosome 2, independently, and (ii) between two additional QTLs on chromosome 3. In addition, we observed that selection against allele(s) from the parental suppressive line, mainly on chromosome 2, occurred when building the RILs in a driver-free background. This suggests that some autosomal alleles inherited from the suppressive line showed deleterious fitness effects when the parental genomes came into contact.

\section{Materials and methods}

\section{Fly stocks}

All stocks are kept on axenic medium (David 1962) at $18^{\circ}$ C. The parental lines that have been used for the RIL production are ST8 and SR (Fig. 1a). The other lines were used to test the suppression ability of those RILs (Fig. 1b).

The ST8 line is our reference standard line, free of driver and suppressor (Montchamp-Moreau and Cazemajor 2002).

The $S T 8 / C(1) R M, y w$ line is derived from the reference standard line. In this line females carry the compound $\mathrm{X}$ chromosomes C(1)RM,yw while all other chromosomes come from the ST8 line.

The $S R$ line is the reference Paris SR line, which carries $\mathrm{X}^{\mathrm{SR}}$ chromosomes and shows complete autosomal suppression (Cazemajor et al. 1997).

The ST8;Cy;Delta/Ub line carry sex chromosomes from the ST8 line and chromosomes 2 and 3 with the dominant visible mutations $\mathrm{Cy}$ and Ubx/Delta, respectively (Montchamp-Moreau and Cazemajor 2002)

The XSR6 line was used to reintroduce the driver in all RILs for assessing their suppression strength. In this line, the males carry the reference $\mathrm{X}^{\mathrm{SR} 6}$ chromosome from the SR line and the females carry the compound X C(1)RM,yw. The Y chromosome and the autosomes are from the ST8 line (Fouvry et al. 2011).

\section{RIL production}

In order to prevent any positive selection of suppressing alleles throughout the crossing procedure the initial cross for producing the RILs was performed between two driverfree inbred lines: females from the suppressor-free ST8/C(1) RM,yw line were mated with males carrying sex chromosomes from the standard ST8 line and suppressing autosomes from the SR line.

The crossing scheme used to produce the RILs is described in Fig. 1, it was conducted at $25^{\circ} \mathrm{C}$. First, in order to generate recombination events, large mass crosses (around 2000-3000 flies at each generation) were performed during six generations. Then, 600 lines were started by mating pairs of F7 flies, and full-sib pair matings were carried out during at least 16 generations. The resulting RILs had the following genome: $\mathrm{X}^{\mathrm{ST} 8}$ (males) or a compound $\mathrm{X}$ chromosome (females), a standard Y chromosome $\left(\mathrm{Y}^{\mathrm{ST}}\right.$ ) and mosaic autosomes between the ST8 (non-suppressing) and SR (suppressing) lines.

\section{Phenotyping (suppression ability)}

The ability of the RIL autosomes to suppress the drive was measured after replacing the $\mathrm{X}^{\mathrm{ST} 8}$ chromosomes of males by the reference chromosome $\mathrm{X}^{\mathrm{SR} 6}$. This was completed by using females carrying the C(1)RM,yw $\mathrm{X}$ chromosomes (see the crossing scheme in Fig. 1).

Then, for each RIL, males carrying $X^{\text {SR6 }}$ were individually mated, with standard ST8 females. Crosses were carried out at $25^{\circ} \mathrm{C}$, flies were transferred into new vials 4 days later. Offspring were sexed until no more flies emerged and only crosses producing at least 50 offspring were taken into account. For each RIL the sex ratio was measured for 10 individual crosses at least.

\section{DNA sequencing and genotyping}

DNA of parental lines was extracted from the whole body, using a classical extraction protocol with phenol-chloroform, and sequenced using Illumina Hiseq 2500 pair ends technology (http://www.illumina.com/). Read alignments were performed using GSNAP (Wu and Nacu 2010) and SAMtools (Li et al. 2009). SNPs were called with GATK (McKenna et al. 2010) and 17,000 selected SNPs were tested in silico by the LGC Genomics company for their compatibility with the $\mathrm{KASP}^{\mathrm{TM}}$ technology. We estimated the genetic position of each SNP according to its physical position in the Drosophila melanogaster genome and the genetic map produced by Attrill et al. (2016), taking into account the inversion on the third chromosome between $D$. simulans and D. melanogaster (Mahowald and Kambysellis 1980). A final list of 
Fig. 2 Frequency distribution of the ST8 SNP alleles along chromosomes 2 and 3 in the RIL population. Each circle represents one marker. Solid circles correspond to the markers that showed highly significant segregation bias $(P$ value $<10^{-15}$ ). Markers are ordered along the $X$-axis according to their genetic position. The $Y$-axis indicates the frequency of the ST8 allele

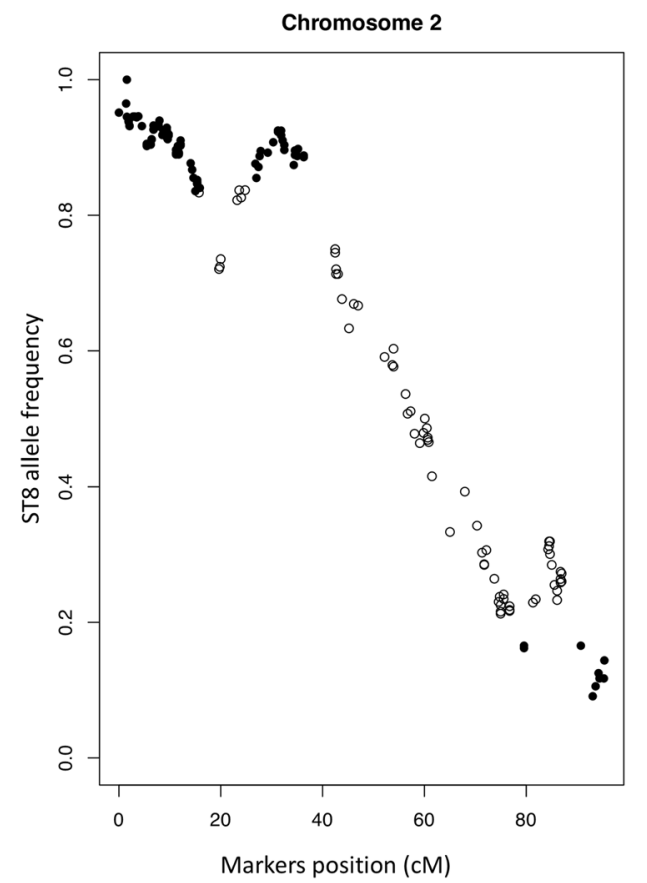

384 SNPs was then established, which were evenly distributed along the genome according to their position on the genetic map.

Adult flies from every RIL were kept at $-80{ }^{\circ} \mathrm{C}$ until DNA extraction. DNA was extracted from the whole body of 15 flies per RIL using a classical protocol with isopropanol precipitation. $3 \mu \mathrm{g}$ of genomic DNA were used for genotyping.

The RILs were genotyped by the LGC genomics company (http://www.lgcgenomics.com/) using the $\mathrm{KASP}^{\mathrm{TM}}$ technology (Semagn et al. 2014).

\section{Genetic map construction}

The genetic map was built using the R/qtl package (Broman et al. 2003). First, genotype and phenotype data were imported in R/qtl using the read.cross function. The cross object was set as a 'RIL sibling' cross type. Since residual heterozygosity cannot be taken into account with RILs, heterozygous calls were considered as missing data. Using the subset function, five RILs with less than 200 genotyped SNPs were discarded. We tested each marker locus for Mendelian segregation of alleles with a $X^{2}$-test using the geno.table function, which allowed to identify 134 markers in distortion $\left(P\right.$-value $\left.<10^{-15}\right)$. The distribution of allele frequencies along the RIL chromosomes indicated a real segregation distortion rather than genotyping errors (see Fig. 2). We chose to keep all markers for the analyses and use the markerlrt function, which takes account of the segregation distortions to estimate the recombination fractions between markers. Eventually 152 RILs and 373 markers were kept. The orders of local markers were checked with the ripple function, using a maximumlikelihood method that compare the likelihoods of different orders. Finally, inter-marker distances on the genetic map were re-estimated with the Kosambi function using the est.map function. We obtained a map with a total length of $96.58 \mathrm{cM}$ for chromosome 2 and $172 \mathrm{cM}$ for chromosome 3 . The value for chromosome 3 was slightly higher than the known size of this chromosome in D. simulans (True et al. 1996). This is expected in the context of highly RILs resulting from advanced intercrosses, which artificially increase the size of the genetic map. On the other hand, the segregation distortion on chromosome 2 lowers the recombination fraction and consequently the genetic distance between markers. We checked for the collinearity of our genetic map with the genome sequence, plotting physical versus genetic positions for each marker (Supplemental Fig. 1).

\section{QTL mapping}

We used the qqplot and qqnorm $\mathrm{R}$ functions to test for normality of the sex-ratio phenotype distribution. The original phenotypic variable (female proportion) was transformed through a logit function, then we calculated the mean for each RIL line. The resulting transformed variables were used as phenotypes for QTL mapping.

QTL mapping was performed with the R/qtl package. First, a single QTL analysis was performed using the 
Fig. 3 Variation of SR autosomal suppression among the RILs: Distribution of the percentage of females in the progeny of males carrying $\mathrm{X}^{\mathrm{SR} 6}$ and autosomes of each RILs. The 152 RILs are classified along the $X$-axis in increasing order of the mean percentage of female in the progenies (black line). Each boxplot represents one RIL, the limits of the box correspond to the 10th and 90th percentiles, the median is indicated by a black bar. The dotted gray line marks the equal sex ratio $(0.5)$ and the solid gray line the mean female percentage in the progeny of males carrying $\mathrm{X}^{\mathrm{SR6}}$ in a suppressor-free background (0.95)

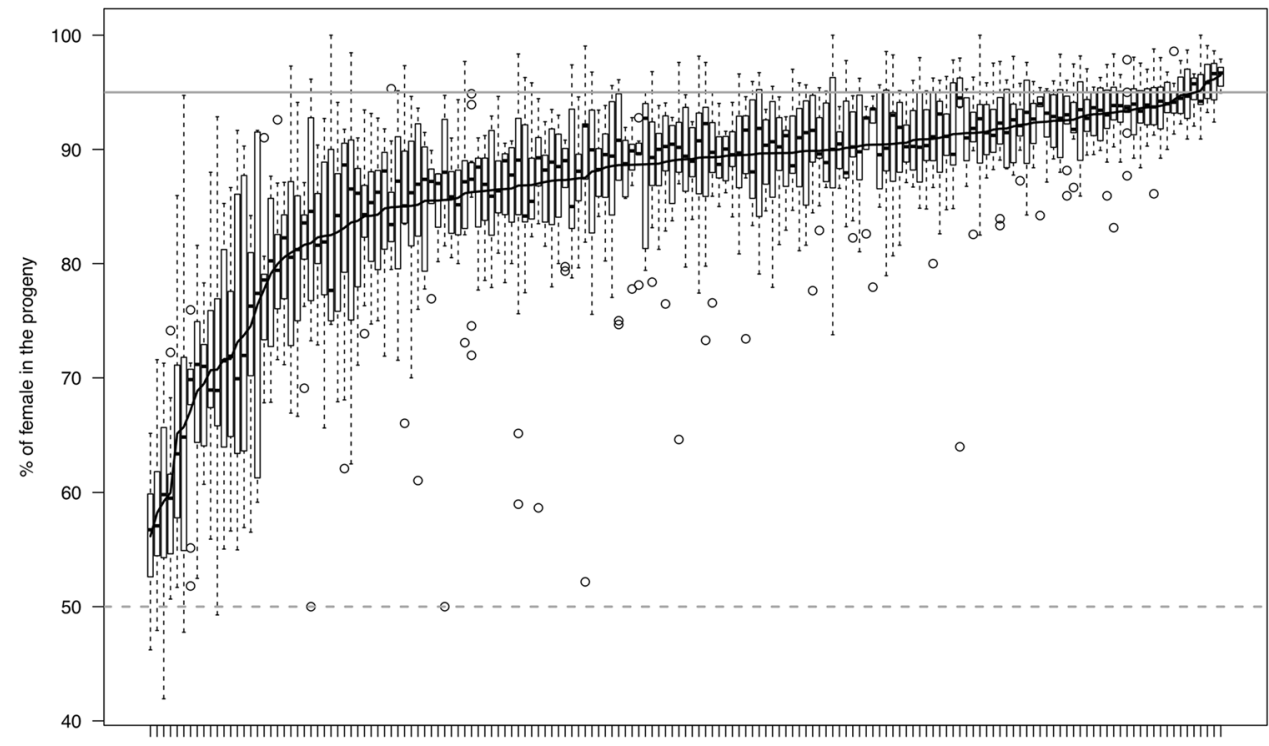

RIL
Haley-Knott regression method with the scanone function. Then, taking interacting loci into account, a two-QTL scan analysis was performed with the scantwo function, also using the Haley-Knott regression method. A permutation test with 1000 replicates (Churchill and Doerge 1994) allowed to estimate the genome-wide LOD threshold corresponding to a 5\% significance level. Finally, the identified QTLs were incorporated into the analysis using a multiple QTL model. The model was first built with the makeqtl function that takes account of interactions, then tested with the fitqt function using the Haley-Knott regression method. The refineqtl function was finally used to re-estimate the position of the QTL by maximizing the LOD score. Once the model was fixed, the functions addqtl, addint, and addpaire were used to look for additional QTLs or interactions. To test for epistasis between QTLs, the model with epistatic interactions was compared to the additive model. We considered epistasis as significant when having a LOD difference $>2$. The LOD score, the percentage of variance explained by the QTL, the number of individuals per genotypic class, the ANOVA Fisher's $F$-value, the additive effect of each QTL and of each QTL*QTL interaction were computed through the fitqtl function. In order to assess the percentage of variance explained by each QTL individually and the interactions, we ran the analysis with different models with and without including the interactions.

For each QTL position, the lodint function was used to estimate 2-LOD confidence intervals. Markers localized at the ends of the intervals were identified using the findmarker function and their physical position was determined.

The representation of the phenotypic effect of each QTL was generated with the effectplot function.

\section{Results}

\section{Segregation distortion during RIL production}

Among the 600 RILs started at the end of the recombination phase, only 152 survived the inbreeding phase during 16 generations. This indicates a low fitness of the lines, which was not unexpected. The 152 surviving RILs were genotyped and phenotyped for their suppression strength. Among them $132(87 \%)$ show weak or no suppression ability (Fig. 3), with more than $80 \%$ of females on average in the progeny. Indeed the mean female percentage distribution is centered around $90 \%$. Only six RILs show a strong suppression phenotype ( $<66 \%$ females), suggesting that very few RILs inherited a full set of suppressors, as expected if suppression is determined by multiple locus and/or epistatic interactions.

However, the segregation of the parental alleles from the suppressing SR and non-suppressing ST8 lines among the RILs is not random (Fig. 2). Among the 373 autosomal SNPs examined, a subset of 134, of which 114 are on chromosome 2 , shows a highly significant segregation bias ( $\chi_{-1}^{2}, p$-value $\left.<1.9 * 10^{-15}\right)$. Many RILs are identical over a large portion of chromosome 2, with only one major recombination event. Most of the markers on the $2 \mathrm{~L}$ arm show high frequencies of ST8 alleles (90\%). In the same way, the ST8 alleles predominate in several region of chromosome 3 . On the opposite, the markers in the distal part of the 2R arm show extremely low frequencies of ST8 alleles (around 10\%). Such a distortion cannot be due to genotyping errors but rather suggests a counter-selection of deleterious alleles from the SR or ST autosomes, depending 
Fig. 4 LOD profiles for the fiveQTL model of suppression ability. The markers are plotted along the horizontal axis, according to their genetic position on each chromosome (cM). The corresponding LOD scores come from the

Haley-Knott regression analysis and include the interaction effect

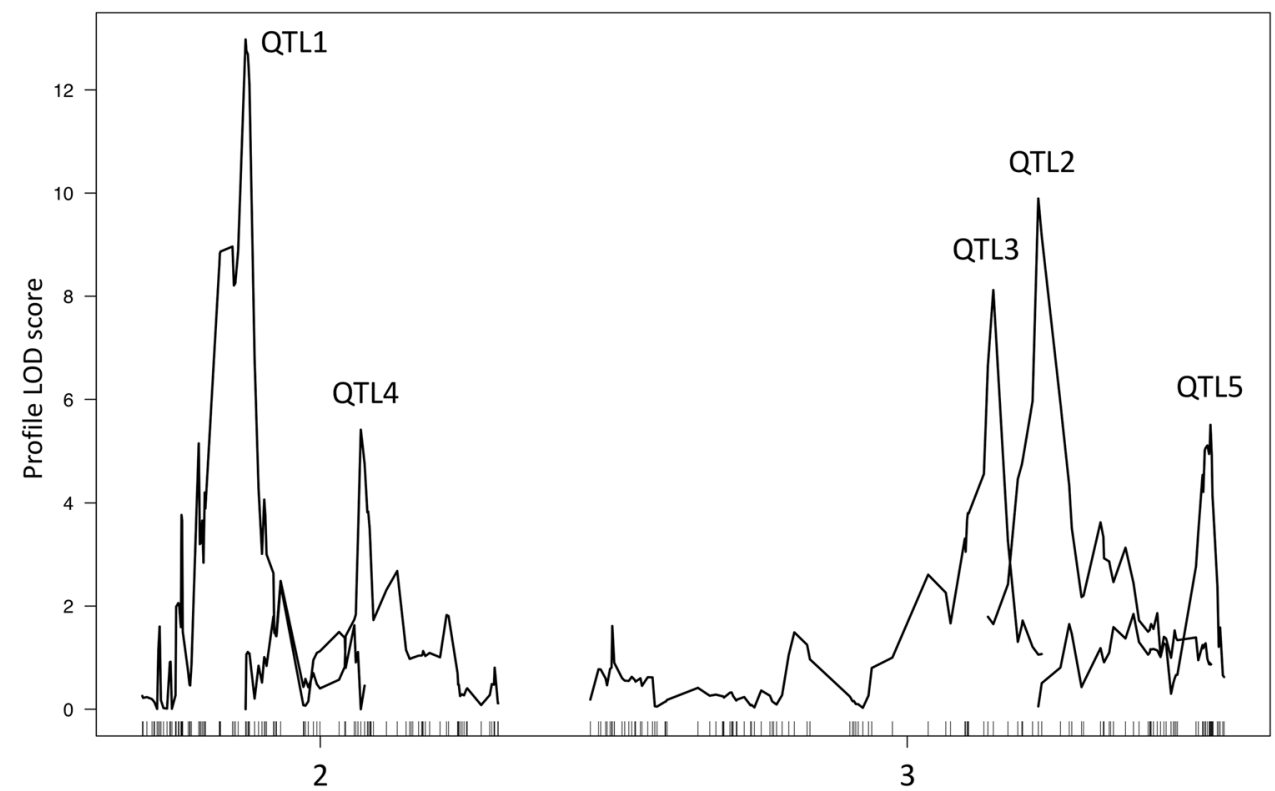

of the regions, and possibly negative epistasis between ST and SR alleles among loci on chromosome 2.

\section{Drive suppression involves at least five loci and epistatic interactions}

First, the single QTL analysis by Haley-Knott regression detected a QTL on chromosome 2 (QTL1) and chromosome 3 (QTL2). Those QTLs are significant at a 5\% threshold after 1000 permutations (threshold at 2.63) with a LOD score of 5.93 and 3.47 , respectively.

In order to look for interacting QTLs, we performed a two-dimensional QTL analysis. We detected an additive interaction between QTL2 and an additional QTL on chromosome 3 (QTL3), with a LOD score of 6.71 (the threshold after permutation was at 3.98 for additive interaction). Furthermore, an epistatic interaction between QTL1 and 2 was detected with a LOD score of 11.27 (the threshold after permutation was at 5.64 for the epistatic interaction). Finally, using a multiple QTL approach, we tested a full model starting with the three QTLs and the epistatic interactions (sex ratio $=\mu+\mathrm{Q} 1+\mathrm{Q} 2+\mathrm{Q} 1 * \mathrm{Q} 2+$ Q3). This model had a LOD score of 13.16 and explained $32.9 \%$ of total variance. When fitting this model, we found two additional QTLs (QTL4, LOD score 5.42 and QTL5, LOD score 5.51) on chromosome 3 and chromosome 2, respectively. QTL5 is in epistatic interaction with QTL3 with a LOD score of 4.09 and QTL4 is also in epistatic interaction with QTL2, with a LOD score of 4.94. The final model $\quad($ sex $\quad$ ratio $=\mu+\mathrm{Q} 1+\mathrm{Q} 2+\mathrm{Q} 3+\mathrm{Q} 4+\mathrm{Q} 5+$ $\mathrm{Q} 1 * \mathrm{Q} 2+\mathrm{Q} 2 * \mathrm{Q} 4+\mathrm{Q} 3 * \mathrm{Q} 5)$ gives a $\mathrm{LOD}$ score of 24.18 and explains $51.93 \%$ of the sex-ratio phenotypic variance in the RIL population (Fig. 4). When testing the effect of each
QTL alone without the interactions, we showed that QTL1 and QTL3 had a significant effect alone (Table 1). The effects of the three other QTLs were only due to their interactions with other loci (Table 1).

Unsurprisingly, all single QTLs show a decrease of the sex-ratio bias associated with the suppressing SR allele (Fig. 5a and Table 1). The suppression effect of the interactions between pairs of QTL, Q1+Q2+Q1*Q2 and $\mathrm{Q} 2+\mathrm{Q} 4+\mathrm{Q} 2 * \mathrm{Q} 4$, is higher than expected from the additive effects of each single QTL, which is typical of positive epistasis (Fig. 5b). On the opposite, the interaction between QTL3 and QTL5 (Q3+Q5+Q3*Q5) is negative compared to the single effect of QTL3 and QTL5 independently (Fig. 5c). The QTL3 SR allele decreases the sexratio bias in the absence of the QTL5 SR allele but has no effect in its presence, and reciprocally. This suggests antagonistic epistasis between those QTLs.

In order to determine the physical size of each region of interest, we computed the 2-LOD confidence interval of each QTL, using the physical position of markers flanking the interval (Table 1). The confidence intervals span from 0.83 to $4.3 \mathrm{Mb}$ depending on the QTL and contain between 125 and 567 genes, which hinders the identification of candidate genes at this stage.

\section{Discussion}

Our genetic mapping experiment identified five QTLs involved in the autosomal suppression of Paris SR drive: two QTLs on chromosome 2 and three on chromosome 3, all showing epistatic interactions. Our results are consistent with those of a first genetic analysis performed by 
Table 1 Multiple QTL model for the suppression ability

\begin{tabular}{|c|c|c|c|c|c|c|c|c|c|}
\hline QTL & $\mathrm{Chr}$ & Physical interval & $\begin{array}{l}\text { Genetic localization } \\
(\mathrm{cM})^{\mathrm{a}}\end{array}$ & LOD & $\% \operatorname{var}^{\mathrm{b}}$ & $\begin{array}{l}F \text {-value }(P- \\
\text { value })\end{array}$ & $\begin{array}{l}\text { Estimated effect } \\
(\mathrm{SE})\end{array}$ & $\begin{array}{l}\text { Number of } \\
\text { Ind. }^{d}\end{array}$ & $\begin{array}{l}\text { Number of } \\
\text { genes }\end{array}$ \\
\hline 1 & 2 & $\begin{array}{l}2.8 \mathrm{Mb} \\
\text { 2L: } 16313310 . .19101804\end{array}$ & $25-29$ & 7.206 & 17.286 & $\begin{array}{l}35.62(1.74 \mathrm{e} \\
-08)\end{array}$ & $-0.41(0.052)$ & 16 & 336 \\
\hline 2 & 3 & $\begin{array}{l}0.85 \mathrm{Mb} \\
\text { 3R: } 9884541 . .10738671\end{array}$ & $120-125$ & 1.186 & 2.591 & $5.34(0.022)$ & $-0.25(0.053)$ & 48 & 125 \\
\hline 3 & 3 & $\begin{array}{l}\text { 1.1 Mb } \\
\text { 3R: } 6028677 . .71280680\end{array}$ & $107-112$ & 2.986 & 6.708 & $\begin{array}{l}13.82(2.86 \mathrm{e} \\
-04)\end{array}$ & $-0.04(0.052)$ & 37 & 132 \\
\hline 4 & 2 & $\begin{array}{l}1.14 \mathrm{Mb} \\
\text { 2R: } 11484071 . .12623014\end{array}$ & $57-61$ & 0.144 & 0.31 & $0.64(0.425)$ & $-0.11(0.036)$ & 70 & 149 \\
\hline 5 & 3 & $\begin{array}{l}4.3 \mathrm{Mb} \\
\text { 3R: } 20238565 . .24589803\end{array}$ & $165-170$ & 0.8 & 1.74 & $3.58(0.06)$ & $-0.03(0.047)$ & 27 & 567 \\
\hline $1 * 2$ & - & - & - & 4.413 & 6.875 & $\begin{array}{l}20.45(1.27 \mathrm{e} \\
-05)\end{array}$ & $-0.24(0.053)$ & 7 & - \\
\hline $2 * 4$ & - & - & - & 4.938 & 7.757 & $\begin{array}{l}23.08(3.88 \mathrm{e} \\
-06)\end{array}$ & $-0.18(0.038)$ & 27 & - \\
\hline $3 * 5$ & - & - & - & 4.092 & 6.344 & $\begin{array}{l}18.88(2.63 \mathrm{e} \\
-05)\end{array}$ & $0.21(0.050)$ & 7 & - \\
\hline
\end{tabular}

${ }^{\mathrm{a}}$ Genetics positions are given according to the genetic map constructed with r-qtl

${ }^{b}$ Percentage of phenotypic variance explained. The LOD score and \% var for each single QTL were determined from a different model where interactions were eliminated, in order to test only the effect of a single QTL without the interaction effect

${ }^{c}$ Estimated effect of the suppressive allele (SR6) compared to the standard one (ST8) on the

transformed phenotypic value

${ }^{\mathrm{d}}$ Number of individuals with the SR allele at the corresponding QTLs

Cazemajor et al. (1997) on the same SR line, which had shown that both major autosomes had important suppressor effects at the homozygous state. In their experiment, homozygous loci on chromosomes 2 and 3 decreased the proportion of females in the offspring of males carrying the $X^{\mathrm{SR}}$ by $15 \%$ and $21 \%$, respectively, compared to the heterozygous state, while an equal sex-ratio was restored by their combined effects. Here our model explains $51.93 \%$ of the phenotypic variation observed among the 152 RILs examined, but we cannot exclude that the percentage of variance explained is overestimated through a Beavis effect (Beavis 1994) due to the relatively small RIL sample size, or by segregation distortions in some chromosome regions. This overestimation is expected to be higher for QTL with an actual weak effect. The sample size of individuals contributing to assess the effect of the interactions between QTL1 and QTL2, and between QTL3 and QTL5 is low and we cannot rule out the hypothesis that these effects are overestimated. Nevertheless, since these effects are highly significant, we considered them in the model. The segregation distortion observed on chromosome 2 could have an impact on the estimated position of the QTL, but does not invalidate the presence of QTL 1 with such a strong effect on this chromosome.

The distribution of the sex-ratio phenotypes among the RILs is strongly biased towards non-suppressing lines, with a few percent of the RILs showing nearly complete suppression (see Fig. 3). Given our results, we can postulate that this is at least partly due to the non-additive basis of the quantitative model of suppression. Only lines carrying suppressing alleles at a sufficient number of loci, which potentiate each other through positive epistasis, can have an appreciable suppressor effect. On the other hand, our study has revealed suppressor loci that probably do not contribute to drive suppression in the SR line, such as QTL3 and QTL5, because of negative epistasis.

However, we cannot exclude that more loci are involved in suppression, for several reasons. First, loci in the SR line may have not been revealed by our analysis because they have a weak effect on the phenotype. In this case, only more lines could have helped detecting them. Second, genetic variation could have been lost during RIL production because of strong segregation distortion, especially towards ST8 alleles in the region containing the two main QTLs (QTL1 and QTL2). Suppressing alleles from the SR line could have been strongly counter-selected because they have deleterious fitness effects, either by themselves or by negatively interacting with ST8 alleles at other loci (that would also show segregation distortion, like on chromosome 2). Both sources of deleterious effects are obviously not necessarily mutually exclusive. On the one hand we intentionally produced the RILs in a driver-free context to avoid any positive selection of suppressors. While suppressor alleles have co-evolved with $\mathrm{X}^{\mathrm{SR}}$ chromosomes in 
Fig. 5 Sex-ratio phenotype value for each QTL allele. Effect plot of the main QTL (QTL1) on chromosome 2 (a). Effect plot of the interaction QTL, QTL1*QTL2 (b) and QTL3*QTL5 (c). The $Y$-axis represent the phenotypic value (sex-ratio) after a transformation using logit function
A

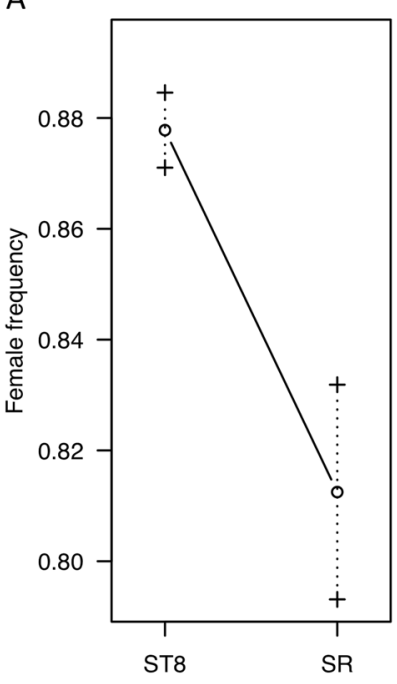

QTL1
B

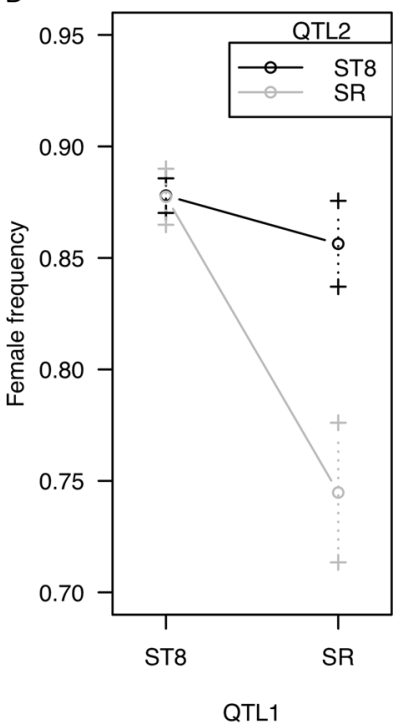

C

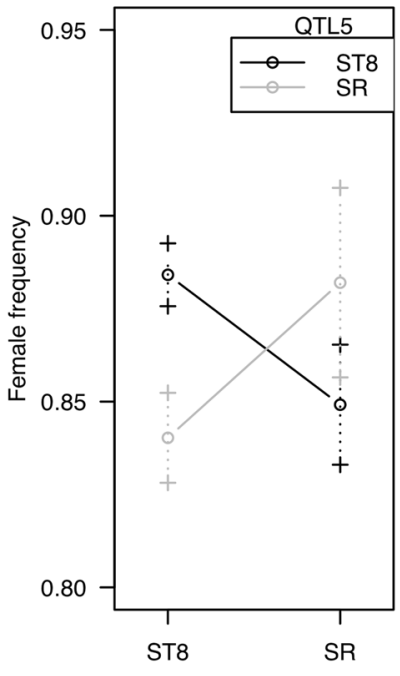

QTL3

natural populations of $D$. simulans, it cannot be excluded that they impose a cost on individual fitness and are counter-selected in a driver-free background. This cost would not have been revealed previously in the lab because autosomal suppression has always been maintained along with an $\mathrm{X}^{\mathrm{SR}}$. On the other hand, the SR and ST8 lines used here came from two different geographic areas, Seychelles and Tunisia, respectively. Genetic incompatibilities was shown to be common between different populations of the same species and can be a source of linkage disequilibrium in RIL genomes (Corbett-Detig et al. 2013; Colomé-Tatché and Johannes 2016). Furthermore, both parental lines have been maintained in the lab for several decades and became highly inbred, facilitating the evolution of allelic incompatibility. All of these phenomena may contribute to the biased distribution of phenotypes among the RILs. Finally, suppressor alleles may occur in D. simulans at other loci than those in the SR line. Autosomal suppressors are regular components of the Paris system in the wild, even in the presence of strong $\mathrm{Y}$ resistance, and have proven to be polymorphic within and between populations (MontchampMoreau et al. 2001; Atlan et al. 2003). Different suppressor alleles may have emerged independently in places remote from each other, and consequently we cannot generalize the results obtained here with the SR strain line derived from flies collected on a Seychelles island. However, the suppressor alleles of the SR line proved to be efficient at the heterozygous state in combination with recessive autosomal suppressor alleles from populations samples as remote as Arabia, New Caledonia, or West indies (Atlan et al. 1997; Montchamp-Moreau et al. 2001). This strongly suggests that the autosomal suppression of Paris SR drive has a comment genetic basis across the species range. In addition, suppressors are suspected to be redundant (Atlan et al.
2003). Redundancy means that there are more loci involved in the suppression than necessary to completely suppress drive. In that way, some of those suppressors could counteract each other, as this seems the case for QTL3 and QTL5.

Suppression in the Paris SR system of $D$. simulans proves here to be extremely complex with multiple loci and interactions involved, which is in sharp contrast with the monogenic suppression reported in this same species for the Winters SR system. In the latter case, the drive is caused by an additional copy of the Dox gene (Tao et al. 2007b), allowing a simple suppression mechanism through gene duplication and RNA interference (Tao et al. 2007a; Lin et al. 2018). In the same way, a driver-suppressor system recently identified in the yeast Saccharomyces kambucha illustrates the diversity of the underlying mechanisms. The particularity of this system is that the same gene, wtf4, acts both as a poison (driver) and an antidote (drive suppressor or resistance). Indeed wtf4 produces two transcripts: a short one that encodes the poison in all gametes and a long one encoding the antidote that is only expressed in the gametes carrying wtf4 (Nuckolls et al. 2017). Drive in the Paris SR system, which involves dysfunctional alleles of the HPID2 gene (Helleu et al. 2016) in epistatic interaction with a component of a tandem duplication, may be much complicated to rescue. This could explain the evolution of multiple mechanisms of compensation involving different suppressor elements.

The multigenic nature of Paris SR drive suppression strikingly recalls the autosomal segregation distorter (SD) system of D. melanogaster. In this system two major loci acting as dominant suppressors were first detected in natural populations (Hiraizumi and Thomas 1984; Trippa and Loverre 1975). However, Lyttle (1979) reported the 
emergence of a polygenic and recessive suppression of SD in an experimental population. The suppressor alleles at multiple loci, each with a small effect on drive, may have pre-existed among the founders or rather have been generated by de novo mutations. Finally, those suppressors proved to be different from the ones from natural populations, favouring the hypothesis that suppression can occur through a variety of ways and evolve rapidly even through a recessive polygenic basis. Even though the molecular mechanisms underlying SD drive remains unclear, several arguments are in favor of the regulation of heterochromatin via small RNA-mediated processes (Larracuente and Presgraves 2012). Is it coincidental that one of the Paris SR drivers, HP1D2, is a member of the HP1 gene family and originates from a duplication of HPID (Rhino) that encodes a key factor in the piRNA cluster transcription (Zhang et al. 2014)? HP1D2 specifically binds the heterochromatic Y chromosome in pre-meiotic male germ cells (Helleu et al. 2016). The driving alleles of $H P 1 D 2$ act epistatically with a component of a short segmental duplication $\left(D p^{S R}\right)$, yet to be identified. But strikingly, $D p^{S R}$ contains a truncated copy of $\operatorname{Trf} 2$, potentially encoding a short Trf2 isoform (Fouvry et al. 2011), which has recently been shown to be recruited to Rhino-decorated pi-RNA source loci in D. melanogaster (Andersen et al. 2017).

The finding that the driver alleles of HPID2 are dysfunctional variants strongly suggests that Paris SR drive results from a disruption of heterochromatin regulation. We can reasonably think that the normal segregation of the $\mathrm{Y}$ chromosome is restored through compensating heterochromatin regulation factors. If autosomal suppressors are, like the driver itself, involved in heterochromatin regulation, then the multiplicity of genes contributing to this process could explain the polygenic nature of autosomal suppression.

\section{Disclaimer}

We certify that none of the material we present in this manuscript has been published or is in under consideration for publication elsewhere.

\section{Data availability}

File S1 contains phenotypic and genotypic data for all individuals. The QTL mapping script is provided in File S2, in.R format. File S3 contains the plot of physical versus genetic positions for each marker. RILs genotypes and genetic position for markers available from Dryad: https:// doi.org/10.5061/dryad.5dk2fs5.

Acknowledgements We thank Sylvie Nortier for the preparation of fly medium. We thank Rim Berdkan and Romuald Laso-Jadart for their help to handle flies during the experiment. We also thank Anne Génissel and Quentin Helleu for helpful discussions. This work was supported by the Agence Nationale de la Recherche (Grant ANR-12BSV7-0014-01). PRG was funded by the ANR grant and CC is funded by a French ministerial Ph.D. scholarship.

\section{Compliance with ethical standards}

Conflict of interest The authors declare that they have no conflict of interest.

\section{References}

Andersen PR, Tirian L, Vunjak M, Brennecke J (2017) A heterochromatin-dependent transcription machinery drives piRNA expression. Nature 549:54-59

Atlan A, Merçot H, Landre C, Montchamp-Moreau C (1997) The sexratio trait in Drosophila simulans: geographical distribution of distortion and resistance. Evolution 51:1886-1895

Atlan A, Capillon C, Derome N, Couvet D, Montchamp-Moreau C (2003) The evolution of autosomal suppressors of sex-ratio drive in Drosophila simulans. Genetica 117:47-58

Attrill H, Falls K, Goodman JL, Millburn GH, Antonazzo G et al. (2016) FlyBase: establishing a gene group resource for Drosophila melanogaster. Nucleic Acids Res 44:786-792

Beavis WD, D. B. Wilkinson (ed.) (1994) The power and deceit of QTL experiments: lessons from comparative QTL studies. In: Proceedings of the 49th annual corn \& sorghum industry research conference. American Seed Trade Association, Washington, DC, pp. 250-266.

Broman KW, Wu H, Sen Ś, Churchill GA (2003) R/qtl: QTL mapping in experimental crosses. Bioinformatics 19:889-890

Burt A, Trivers R (2006) Genes in conflict: the biology of selfish genetic elements. Belknap Press of Harvard University Press, Cambridge, MA

Carvalho AB, Klaczko LB (1993) Autosomal suppressors of sex-ratio in Drosophila mediopuncta. Heredity 71:546-551

Cazemajor M, Landre C, Montchamp-Moreau C (1997) The sex-ratio trait in Drosophila simulans: genetic analysis of distortion and suppression. Genetics 147:635-642

Cazemajor M, Joly D, Montchamp-Moreau C (2000) Sex-ratio meiotic drive in Drosophila simulans is related to equational nondisjunction of the Y chromosome. Genetics 154:229-236

Churchill GA, Doerge RW (1994) Empirical threshold values for quantitative trait mapping. Genetics 138:963-971

Clark AG (1987) Natural selection and Y-linked polymorphism. Genetics 115:569-577

Colomé-Tatché M, Johannes F (2016) Signatures of Dobzhansky-Muller incompatibilities in the genomes of recombinant inbred lines. Genetics 202:825-841

Corbett-Detig RB, Zhou J, Clark AG, Hartl DL, Ayroles JF (2013) Genetic incompatibilities are widespread within species. Nature 504:135-137

David J (1962) A new medium for rearing Drosophila in axenic condition. Drosoph Inf Serv 36:128

Fisher RA (1930) The genetical theory of natural selection.. Oxford University Press, Oxford

Fouvry L, Ogereau D, Berger A, Gavory F, Montchamp-Moreau C (2011) Sequence analysis of the segmental duplication responsible for paris sex-ratio drive in Drosophila simulans. G3 $1: 401-410$

Hamilton WD (1967) Extraordinary sex ratios. Science 156:477-488

Helleu Q, Gérard PR, Montchamp-Moreau C (2015) Sex chromosome drive. Cold Spring Harb Perspect Biol 7:a017616 
Helleu Q, Gérard PR, Dubruille R, Ogereau D, Prud'homme B et al. (2016) Rapid evolution of a Y-chromosome heterochromatin protein underlies sex chromosome meiotic drive. PNAS 113:4110-4115

Hiraizumi Y, Thomas AM (1984) Suppressor systems of segregation distorter (SD) chromosomes in natural populations of Drosophila melanogaster. Genetics 106:279-292

Hurst LD, Pomiankowski A (1991) Causes of sex ratio bias may account for unisexual sterility in hybrids: a new explanation of Haldane's rule and related phenomena. Genetics 128:841-858

Jaenike J (2001) Sex chromosome meiotic drive. Annu Rev Ecol Syst 32:25-49

Jaenike J (1999) Suppression of sex-ratio meiotic drive and the maintenance of Y-chromosome polymorphism in Drosophila. Evolution 53:164-174

Jutier D, Derome N, Montchamp-Moreau C (2004) The sex-ratio trait and its evolution in Drosophila simulans: a comparative approach. Genetica 120:87-99

Keais GL, Hanson MA, Gowen BE, Perlman SJ (2017) X chromosome drive in a widespread Palearctic woodland fly, Drosophila testacea. J Evol Biol 30:1185-1194

Larracuente AM, Presgraves DC (2012) The selfish segregation distorter gene complex of Drosophila melanogaster. Genetics 192:33-53

Li H, Handsaker B, Wysoker A, Fennell T, Ruan J et al. (2009) The sequence alignment/map format and SAMtools. Bioinformatics 25:2078-2079

Lin CJ, Hu F, Dubruille R, Vedanayagam J, Wen J, Smibert P, Loppin B, Lai EC (2018) The hpRNA/RNAi pathway is essential to resolve intragenomic conflict in the Drosophila male germline. Dev Cell 46:316-326.e5

Lyttle TW (1979) Experimental population genetics of meiotic drive systems II. Accumulation of genetic modifiers of segregation distorter (SD) in laboratory populations. Genetics 91:339-357

Lyttle TW (1991) Segregation distorters. Annu Rev Genet 25:511-581

Mahowald AP, Kambysellis MP (1980). Oogenesis. In: Ashburner M, Wright TRF (eds.) The genetics and biology of Drosophila. Academic Press, London, pp. 141-224.

McKenna A, Hanna M, Banks E, Sivachenko A, Cibulskis K et al. (2010) The Genome Analysis Toolkit: a map reduce framework for analyzing next-generation DNA sequencing data. Genome Res 20:1297-1303

Meiklejohn CD, Tao Y (2010) Genetic conflict and sex chromosome evolution. Trends Ecol Evol 25:215-223

Montchamp-Moreau C, Ginhoux V, Atlan A (2001) The Y chromosomes of Drosophila simulans are highly polymorphic for their ability to suppress sex-ratio drive. Evolution 55:728-737
Montchamp-Moreau C, Cazemajor M (2002) Sex-ratio drive in Drosophila simulans: variation in segregation ratio of $\mathrm{X}$ chromosomes from a natural population. Genetics 162:1221-1231

Montchamp-Moreau C, Ogereau D, Chaminade N, Colard A, Aulard S (2006) Organization of the sex-ratio meiotic drive region in Drosophila simulans. Genetics 174:1365-1371

Nuckolls NL, Núñez MAA, Eickbus MT, Young JM, Lange JJ et al. (2017) Wtf genes are prolific dual poison-antidote meiotic drivers. Elife 6:1-22

Presgraves D (2009). Drive and sperm: the evolution and genetics of male meiotic drive. In: Birkhead TR, Hosken D, Pitnick S (eds.) Sperm biology. Academic Press, London, pp. 471-506.

Semagn K, Babu R, Hearne S, Olsen M (2014) Single nucleotide polymorphism genotyping using Kompetitive Allele Specific PCR (KASP): overview of the technology and its application in crop improvement. Mol Breed 33:1-14

Stalker HD (1961) The genetic systems modifying meiotic drive in Drosophila paramelanica. Genetics 46:177-202

Tao Y, Hartl DL, Laurie CC (2001) Sex-ratio segregation distortion associated with reproductive isolation in Drosophila. Proc Natl Acad Sci USA 98:13183-13188

Tao Y, Masly JP, Araripe L, Ke Y, Hartl DL (2007a) A sex-ratio meiotic drive system in Drosophila simulans. I: an autosomal suppressor. PLoS Biol 5:2560-2575

Tao Y, Araripe L, Kingan SB, Ke Y, Xiao H et al. (2007b) A sex-ratio meiotic drive system in Drosophila simulans. II: an X-linked distorter. PLoS Biol 5:e293

Trippa G, Loverre A (1975) A factor on a wild third chromosome (IIIRa) that modifies the Segregation Distortion phenomenon in Drosophila melanogaster. Genet Res 26:113-125

True JR, Mercer JM, Laurie CC (1996) Differences in crossover frequency and distribution among three sibling species of Drosophila. Genetics 142:507-523

Unckless RL, Larracuente AM, Clark AG (2015) Sex-ratio meiotic drive and Y-Linked resistance in Drosophila affinis. Genetics 199:831-840

Vaz SC, Carvalho AB (2004) Evolution of autosomal suppression of the sex-ratio trait in Drosophila. Genetics 166:265-277

Wu TD, Nacu S (2010) Fast and SNP-tolerant detection of complex variants and splicing in short reads. Bioinformatics 26:873-881

Zhang L, Sun T, Woldesellassie F, Xiao H, Tao Y (2015) Sex ratio meiotic drive as a plausible evolutionary mechanism for hybrid male sterility. PLoS Genet 11:e1005073

Zhang Z, Wang J, Schultz N, Zhang F, Parhad SS et al. (2014) The HP1 homolog Rhino anchors a nuclear complex that suppresses piRNA precursor splicing. Cell 157:1353-1363 